\title{
0816 RISK ANALYSIS PLATFORM FOR RAILWAY TRAFFIC SAFETY
}

E Schnieder, R Slovak, S Wegele* Correspondence: Technical University of Braunschweig/IVA, Langer Kamp 8 D-38106, Braunschweig 38106, Germany

\subsection{6/ip.2010.029215.816}

The risk analysis platform at the Federal Office of Transport in Switzerland contains about 50 safety topics assigned to over 300 railway lines. Occurrence rates and damages have been estimated by experts during the last 5 years. In cooperation with the institute for traffic safety and automation engineering a prototype for risk estimations is currently under development. The main difference to the former approach is the usage of physical models for prediction of accidents and usage of history information's for estimation of the damage size. For some topics (eg, avalanches, ignoring of shunting signals) a Monte-Carlo-Simulation is used to estimate occurrence rates and accident parameter, for example, collision speed, number of involved passengers, danger goods etc. The main purpose of the prototype is the validation of the risk estimation method for the entire Switzerland. In the article we will show three representative examples of implemented risk topics including risk models. The simulation results basing on a subnetwork of $90 \mathrm{~km}$ will be presented. 